\title{
Resiliência psicológica: fator de proteção para idosos no contexto ambulatorial
}

\author{
Psychological resilience: protector factor for elderly assisted in ambulatory
}

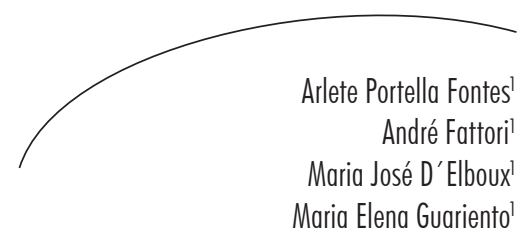

\section{Resumo}

Introdução: Em idosos, a resiliência psicológica associa-se a depressão, saúde física, atividades da vida diária e saúde percebida. Objetivo: Descrever as relações entre funcionalidade, sintomatologia depressiva e cognição em grupos de idosos resilientes e não resilientes. Método: Estudo descritivo, com 59 pacientes do Ambulatório de Geriatria/HC-Unicamp, idade 69-91 anos. Instrumentos: escalas de atividades básicas e instrumentais da vida diária (ABVD, AIVD), sintomas depressivos (EDG), Miniexame do Estado Mental (MEEM) e escala de resiliência. Foram feitas medidas de frequência, posição e dispersão, comparações entre variáveis categóricas (Qui-quadrado e Exato de Fisher) e numéricas (teste de Mann-Whitney, Krushal-Wallis), correlações entre variáveis e análise multivariada de regressão linear. Resultados: Maioria mulheres (80,4\%), 70-89 anos, renda $\leq 2$ salários mínimos, 43,1\% analfabetos, 57\% apresentaram AIVD preservadas (independência em seis ou sete itens); $43 \%$ não preservadas (ajuda total ou parcial em ao menos dois itens); $31,4 \%$ apresentaram sintomatologia depressiva sugestiva de depressão. Os mais resilientes ( $>66 \mathrm{em} 75$ pontos) apresentaram média de 5,2 $\pm 2,1$ AIVD, em relação aos menos resilientes $(3,6 \pm 2,4 ; \mathrm{p}=0,017)$. Os menos resilientes apresentaram média de $6,4( \pm 4,2)$ sintomas depressivos, os mais resilientes média de 2,6( $\pm 2,6 ; p=0,001)$. Correlação negativa entre resiliência e sintomatologia depressiva $(r=-0,688 ; \mathrm{p}<0,01)$. Idosos com frequência $>5$ sintomas depressivos tenderam a apresentar baixos escores em resiliência (análise de regressão linear multivariada). Conclusão: A resiliência constitui importante fator de proteção para idosos ambulatoriais com relativo grau de dependência e evidência de sintomas depressivos.

\footnotetext{
Universidade Estadual de Campinas. Programa de Pós-graduação em Gerontologia, Faculdade de Ciências Médicas. Campinas, SP, Brasil.

Palavras-chave: Resiliência Psicológica. Funcionalidade. Sintomas Depressivos. Idoso. 


\section{Abstract}

Introduction: Among elderly, psychological resilience is associated with depression, physical health, daily living activities and self-perceived health. Objective: To describe the relationships between functionality, depressive symptomatology and resilience between resilient and non resilient groups. Method: Descriptive study, 59 patients from the Geriatric Ambulatory/HC Unicamp, age 69-91 years. Instruments: scales of activities of daily living (ADL), instrumental activities of daily living (IADL), depressive symptoms (GDS), Mini-Mental Exam (MEEM) and resilience. Measurements used were: frequency, position and dispersion, comparisons among categorical variables (Chi-square and Fisher Exact), numerical variables (Mann-Whitney, Krushal-Wallis), co-relations among variables and linear regression multivariate analysis. Results: Mostly women (80.4\%), 70-89 years, income $\leq 2$ minimum wage, $43.1 \%$ illiterate, $57 \%$ showed preserved IADL (independence in six or seven items), $43 \%$ not preserved (partial or total help in at least 2 items), 31.4\% showed depressive symptomatology suggestive of depression. The most resilient ( $>66$ within 75 scores) showed average of 5.2( \pm 2.1$)$ IADL, in relation to the least resilient $(3.6 \pm 2.4 ; \mathrm{p}=0.017)$. The least resilient showed average of $6.4( \pm 4.2)$ depressive symptoms, the most resilient showed average of $2.6( \pm 2.6 ; \mathrm{p}=0.001)$. Negative co-relation between resilience and depressive symptomatology $(\mathrm{r}=-0.688$; $\mathrm{p}<0.01)$. Elderly with frequency $>5$ depressive symptons tended to show low scores in resilience (linear regression multivariate analysis). Conclusion: Resilience is an important protector factor for elders assisted in ambulatory, with a relative degree of dependency and evidence of depressive symptoms.
Key words: Psychological Resilience. Functionality. Depressive Symptoms. Elderly.

\section{INTRODUÇÃO}

A depressão é uma condição clínica frequente, variando entre 4,8\% e 14,6\% em idosos da comunidade e atingindo $22 \%$ dos idosos hospitalizados ou institucionalizados. ${ }^{1}$ Em torno de $45 \%$ dos pacientes com doença coronariana, doença de Parkinson, doença cerebrovascular e doença de Alzheimer apresentam depressão grave. $^{2}$ Entretanto, a prevalência de sintomas depressivos, também reconhecida como depressão menor ou subsindrômica, tal como medida pelas escalas de rastreio, tende a se tornar maior. No Brasil, Lebrão \& Laurenti ${ }^{3}$ encontraram prevalência de $18,1 \%$ em idosos participantes do estudo SABE, na cidade de São Paulo; e no estudo PENSA - Estudo dos Processos de Envelhecimento Saudável, ${ }^{4}$ envolvendo 956 idosos da comunidade em Juiz de Fora, Minas Gerais, registrou-se prevalência de $15 \%$.

Resiliência psicológica é definida como fator de proteção em relação às desordens psicóticas, caso em que os indivíduos resilientes seriam possuidores de maior autoestima, autoeficácia, mais habilidades para resolver problemas e maior satisfação com relações interpessoais, ${ }^{5}$ característica de personalidade que modera os efeitos negativos do estresse e promove a adaptação; ${ }^{6}$ integrando conceitos de plasticidade e capacidade de reserva, dentro da abordagem do desenvolvimento ao longo da vida. ${ }^{7}$ Pode ser comparada a um "amplo guarda-chuva" que abrigaria recursos psicológicos para superação de adversidades, dentre eles a autoestima, as competências pessoais e as crenças de controle, ${ }^{8}$ recursos utilizados para adaptação às tarefas do desenvolvimento.?

O termo tem sido associado a indicadores de envelhecimento bem-sucedido, baixa probabilidade de doença relacionada à incapacidade, capacidade cognitiva e funcionalidade física e engajamento com a vida; $;^{10,11}$ boa qualidade de relacionamentos, integração à comunidade, alto uso de enfrentamento adaptativo e enfrentamento relacionado ao desenvolvimento. ${ }^{12}$ 
No âmbito da funcionalidade, resiliência tem sido associada à capacidade de manter-se ativo e não desenvolver sintomas depressivos diante da adversidade. São encontradas correlações entre resiliência, depressão, saúde física e satisfação com a vida; ${ }^{6}$ depressão, incapacidade funcional, apatia e resiliência. ${ }^{13}$ Em idosos a partir de 80 anos, a depressão é altamente correlacionada com apatia, sendo que entre aqueles com menos de 80 anos, os escores de resiliência, apatia e incapacidade contribuíram igualmente para a variabilidade na Escala de Depressão Geriátrica (EDG), com resiliência associando-se ao manejo da vida diária e sintomas depressivos. ${ }^{13}$ Avaliando resiliência, fatores demográficos, condições crônicas, status funcional, atividade física, autoeficácia, sintomas depressivos, saúde percebida e suporte social, Hardy, Contato \& Gil ${ }^{14}$ encontraram resiliência associada a independência nas atividades da vida diária, boa a excelente saúde percebida e poucos sintomas depressivos.

Conhecer como se relacionam e se associam variáveis como depressão e capacidade funcional em pacientes ambulatoriais pode fornecer pistas para atuação de equipe multidisciplinar junto a idosos em serviços de geriatria e gerontologia, quer do ponto de vista de diagnóstico e tratamento, quer para o planejamento de orientações para familiares e cuidadores.

Este estudo objetivou comparar grupos de idosos resilientes e não resilientes de um ambulatório de geriatria em relação a dados sociodemográficos (idade, gênero, escolaridade, renda mensal), funcionalidade (atividades básicas da vida diária - ABVD e atividades instrumentais da vida diária - AIVD), cognição e sintomatologia depressiva.

\section{MÉTODO}

Trata-se de estudo descritivo, cuja coleta dos dados clínicos foi feita com base nos prontuários médicos preenchidos na data do atendimento de 59 pacientes agendados no Ambulatório de Geriatria do Hospital de Clínicas da Universidade
Estadual de Campinas (AG-Unicamp), com cognição preservada, idade de 69 a 91 anos, avaliados no período de 2009 a 2011. Esse ambulatório atende idosos com 60 anos ou mais, referenciados de serviços de saúde da Região Metropolitana de Campinas, como também de outros ambulatórios do Hospital de Clínicas da Unicamp.

Os critérios de inclusão foram: pacientes do ambulatório de geriatria, por ocasião de realização de consulta, com 60 anos e mais, submetidos ao MEEM (Folstein et al. ${ }^{15}$ validado por Lourenço $\left.{ }^{16}\right)$, como medida de rastreio cognitivo. Foram utilizadas como notas de corte $<14$ para os analfabetos; $<18$ em idosos com um a oito anos de escolaridade e $<24$ para escolaridade acima da $8^{\mathrm{a}}$ série, ${ }^{17}$ tendo sido excluídos aqueles que não atingiam a nota de corte. Os instrumentos foram aplicados oralmente pelos profissionais do ambulatório.

A capacidade funcional foi avaliada por meio das escalas de ABVD (Katz et al. ${ }^{18}$ validadas por Lino et al. ${ }^{19}$ ) e AIVD (adaptada de Lawton \& Brod $\left.^{20}\right)$. Os dados foram finalmente categorizados como ABVD preservadas (aquelas executadas com independência em todos os itens avaliados) e não preservadas (aquelas executadas com comprometimento de ao menos uma atividade). Os dados também foram categorizados como AIVD preservadas (aquelas executadas com independência em seis ou sete itens) e não preservadas (aquelas executadas com ajuda total ou parcial em ao menos dois itens do interrogatório).

A sintomatologia depressiva foi avaliada por meio da Escala de Depressão Geriátrica (adaptada de Yesavage et al. ${ }^{21}$ ), com 15 itens, sendo a pontuação igual ou superior a seis correspondente à presença de sintomas depressivos.

A resiliência psicológica foi avaliada por meio da Escala de Resiliência (adaptada por Pesce et al. ${ }^{22}$ ), sendo composta por três fatores: Fator 1 - resolução de ações e valores (15 itens); Fator 2 - independência e determinação (4 
itens); e Fator 3 - autoconfiança e capacidade de adaptação às situações (6 itens). A adaptação brasileira apresenta 25 itens variando de 1 (discordo totalmente) a 7 (concordo totalmente), com escore máximo de 75 pontos. Apresentou bons indicadores de validade de conteúdo (equivalência semântica), de consistência interna $(0,80)$ e de confiabilidade intraobservador. Após uma avaliação operacional da escala, optou-se pela utilização de uma escala de Likert de 3 pontos, sendo 1 (discordo totalmente), 2 (nem concordo, nem discordo) e 3 (concordo totalmente), uma vez que a escalonagem inicial de 7 graus trouxe confusões para a formulação da resposta, provavelmente devido à baixa escolaridade. Para a classificação dos idosos com elevados e baixos escores em resiliência, utilizou-se a pontuação de respostas com ponto de corte da mediana $(\leq 66)$, sendo que resultados $>66$ foram indicativos de escores elevados em resiliência, em comparação com baixos escores em resiliência $(\leq 66)$.

Foram utilizadas medidas de frequência para as variáveis categóricas (idade, gênero, escolaridade, renda mensal e frequência de sintomas de depressão) e estatísticas descritivas para variáveis numéricas (idade, escores de resiliência, sintomas depressivos, MEEM, além de ABVD e AIVD). A comparação entre variáveis categóricas foi feita pelo teste Qui-quadrado ou Exato de Fisher (para valores esperados menores que cinco). As variáveis numéricas foram comparadas por meio do teste de Mann-Whitney (para duas variáveis categóricas) e Krushal-Wallis (para três ou mais variáveis categóricas). As correlações entre variáveis numéricas foram calculadas por meio do coeficiente de correlação de Spearman. Para avaliar a relação entre as variáveis independentes e a variável de desfecho (resiliência), utilizouse a análise multivariada de regressão linear, com critério stepwise para seleção de variáveis. Neste modelo foram incluídas as variáveis: gênero, idade, renda mensal, escolaridade,
ABVD, AIVD, MEEM e EDG. Para os testes estatísticos, adotou-se o nível de significância de $5 \%$, ou seja, $\mathrm{p}<0,05$.

Este trabalho integra o Projeto Temático "Qualidade de vida em idosos: indicadores de fragilidade e bem-estar subjetivo", que foi aprovado pelo Comitê de Ética em Pesquisa da Faculdade de Ciências Médicas da Universidade Estadual de Campinas, sob parecer no. 240/2003. Todos os participantes assinaram o Termo de Consentimento Livre e Esclarecido.

\section{RESULTADOS}

Houve predominância de mulheres com idade de 70 a 89 anos, média de 75,72 anos ( $\mathrm{dp}=7,76)$, idade mínima de 61 anos e máxima de 91 anos. Registrou-se igual proporção $(43,1 \%)$ de idosos analfabetos e com menos de quatro anos de escolaridade. A maioria tinha renda mensal $\geq$ 2 salários mínimos, sendo que $57 \%$ da amostra apresentavam as atividades básicas da vida diária preservadas e quase $43 \%$ tinham algum grau de dependência. Em relação às AIVD, 57\% apresentavam perdas em sua realização. A maioria dos pacientes idosos apresentava pontuação na EDG inferior a 5 , sendo que $31 \%$ dos idosos apresentavam evidências de sintomatologia depressiva, indicativa de possível depressão.

Quando se compararam as variáveis numéricas e os grupos de resiliência baixa e elevada, associações significativas foram encontradas entre resiliência e AIVD, e resiliência e presença de sintomatologia depressiva. Idosos com elevado grau de resiliência apresentaram média de 5,2( $\pm 2,1)$ AIVD preservadas em relação a $3,6( \pm 2,4)$ AIVD preservadas naqueles com baixa resiliência $(\mathrm{p}=0,017)$. Os que tinham baixa resiliência apresentaram, em média, pontuação $6,2( \pm 4,2)$ na EDG, sendo que os de alta resiliência pontuaram $2,6( \pm 2,6 ; \mathrm{p}=0,001)$, conforme a tabela 1. 
Tabela 1. Comparação entre variáveis númericas e grupos de baixa e elevada resiliência. HCUnicamp,Campinas-SP, 2013.

\begin{tabular}{cccc}
\hline \multicolumn{3}{c}{ Resiliência } \\
\hline M(DP) & Baixa & Elevada & Valor p \\
Idade & $74,9( \pm 7,7)$ & $76,5( \pm 7,8)$ & 0,414 \\
AVD & $5,0( \pm 1,7)$ & $5,3( \pm 1,1)$ & 0,733 \\
AIVD & $3,6( \pm 2,4)$ & $5,2( \pm 2,1)$ & $\mathbf{0 , 0 1 7}$ \\
MEEM & $21,8( \pm 4,5)$ & $23,3( \pm 3,8)$ & 0,225 \\
EDG & $6,2( \pm 4,2)$ & $2,6( \pm 2,6)$ & $\mathbf{0 , 0 0 1}$ \\
\hline
\end{tabular}

Comparando-se idosos com elevada e baixa resiliência total, resiliência Fator 1, Fator 2 e Fator 3 com os valores médios de idade, AIVD e EDG foram encontradas associações significativas entre AIVD e resiliência total, AIVD e resiliência Fator 1. Idosos com baixa resiliência total apresentaram média de 3,6( $\pm 2,4)$ AIVD preservadas, em relação a idosos com elevada resiliência com $5,2( \pm 2,1 ; \mathrm{p}=0,017)$. Esta associação permaneceu quando foram comparados resultados em AIVD e resiliência Fator 1. Idosos com baixa resiliência Fator 1 apresentaram média de 3,7( $\pm 2,3)$ AIVD preservadas, em relação a $5,0( \pm 1,4)$ AIVD em idosos com alta resiliência $(\mathrm{p}=0,032)$. Quando comparados os achados de resiliência e sintomatologia depressiva, diferenças significativas foram encontradas entre idosos com elevada e baixa resiliência total, resiliência Fator 1 e resiliência Fator 3. Idosos com baixa resiliência total apresentaram média de $6,4( \pm 4,2)$ pontos na EDG em relação a 2,6( $\pm 2,6) \mathrm{em}$ idosos com alta resiliência total $(\mathrm{p}=0,001)$. Idosos com elevada resiliência Fator 1 apresentaram média de $2,5( \pm 2,5)$ pontos, em relação a $6,6( \pm 4,1) \mathrm{em}$ idosos com baixa resiliência $(p<0,001)$. Em relação à resiliência Fator 3, foram encontrados valores médios de $6,1( \pm 4,6)$ e $3,2( \pm 2,9)$ pontos na EDG em idosos com baixa e elevada resiliência, respectivamente $(\mathrm{p}=0,022)$, conforme apresentado na tabela 2.

Tabela 2. Comparações entre valores médios de idade, AIVD, sintomas depressivos (EDG), fatores de resiliência e resiliência total. HC-Unicamp, Campinas-SP, 2013.

\begin{tabular}{ccccc}
\hline & Resiliência & Idade & AIVD & EDG \\
\hline Fator 1 & baixa & $74,2( \pm 7,5)$ & $\mathbf{3 , 7}( \pm 2, \mathbf{3})$ & $\mathbf{6 , 6}( \pm \mathbf{4 , \mathbf { 1 }})$ \\
& elevada & $77,0( \pm 7,8)$ & $\mathbf{5 , 0}( \pm 1,4)$ & $\mathbf{2 , 5}( \pm 2, \mathbf{5})$ \\
Fator 2 & valor p & 0,16 & 0,032 & $<0,001$ \\
& baixa & $75,0( \pm 7,0)$ & $4,15( \pm 2,3)$ & $5,6( \pm 4,5)$ \\
& elevada & $76,2( \pm 8,3)$ & $4,7( \pm 2,4)$ & $3,4( \pm 3,0)$ \\
Fator 3 & valor p & 0,557 & 0,29 & 0,075 \\
& baixa & $75,81( \pm 8,5)$ & $3,7( \pm 2,4)$ & $\mathbf{6 , 1}( \pm \mathbf{4 , 6 )}$ \\
& elevada & $75,77( \pm 7,3)$ & $4,9( \pm 2,3)$ & $\mathbf{3 , 2} \mathbf{( \pm 2 , 9 )}$ \\
Resiliência & valor p & 0,96 & 0,076 & 0,022 \\
Total & baixa & $74,9( \pm 7,7)$ & $\mathbf{3 , 6}( \pm 2,4)$ & $\mathbf{6 , 4} \mathbf{( \pm 4 , 2 )}$ \\
& elevada & $76,5( \pm 7,7)$ & $\mathbf{5 , 2}( \pm 2, \mathbf{2})$ & $\mathbf{2 , 6}( \pm 2, \mathbf{6})$ \\
& valor p & 0,414 & 0,017 & 0,001 \\
\hline
\end{tabular}


Foram encontradas diferenças significativas quando comparados idosos com elevada e baixa resiliência quanto às variáveis categóricas e presença de sintomatologia depressiva; 23 dos idosos sem sintomas depressivos pontuaram para elevada resiliência, em relação a 12 idosos com baixa resiliência; 13 idosos com sintomas depressivos pontuaram para baixa resiliência, em relação a três com elevada resiliência $(\mathrm{p}=0,002)$. Diferenças significativas também foram encontradas em relação às AIVD, verificandose que 17 daqueles com AIVD preservadas apresentavam alta resiliência, em relação a sete com baixa. Dentre aqueles com perdas em AIVD, 12 apresentavam elevada resiliência, em relação a 20 com baixa resiliência $(p=0,013)$. E dentre aqueles com menor renda, 19 eram idosos com baixa resiliência, em relação a 14 com elevada $(p=0,003)$, segundo a tabela 3 , a seguir.

Tabela 3. Comparações entre variáveis categóricas e grupos de baixa e elevada resiliência. HC-Unicamp, Campinas-SP, 2013.

\begin{tabular}{|c|c|c|c|c|}
\hline & \multicolumn{4}{|c|}{ Resiliência } \\
\hline & & Baixa & Elevada & Valor $\mathrm{p}$ \\
\hline \multirow[t]{2}{*}{ EDG } & Sem sintomas & 12 & 23 & 0,002 \\
\hline & Com sintomas & 13 & 3 & \\
\hline \multirow[t]{2}{*}{ ABVD } & Preservadas & 14 & 14 & 0,296 \\
\hline & Com perdas & 8 & 13 & \\
\hline \multirow[t]{2}{*}{ AIVD } & Preservadas & 7 & 17 & 0,013 \\
\hline & Com perdas & 20 & 12 & \\
\hline \multirow[t]{2}{*}{ Renda } & $\leq 2 \mathrm{SM}$ & 19 & 14 & 0,003 \\
\hline & $>2 \mathrm{SM}$ & 0 & 8 & \\
\hline \multirow[t]{2}{*}{ Genero } & Fem & 20 & 25 & 0,089 \\
\hline & Masc & 8 & 3 & \\
\hline \multirow[t]{3}{*}{ Escolaridade } & Analfabeto & 11 & 11 & 0,706 \\
\hline & $\leq 4$ anos & 9 & 13 & \\
\hline & $>4$ anos & 4 & 3 & \\
\hline
\end{tabular}

Quando se correlacionaram os valores dos escores de resiliência total e as variáveis "idade", "ABVD", "AIVD", "MEEM" e "EDG", observou-se correlação negativa entre resiliência total e o número de sintomas depressivos $(p<0,01)$, ou seja, quanto maiores foram os escores em resiliência total, menor foi a frequência de sintomas depressivos. Observaram-se correlações negativas entre Fatores 1, 2 e 3 e o número de sintomas depressivos $(\mathrm{p}<0,01 ; \mathrm{p}=0,001 ; \mathrm{p}=0,002$, respectivamente), e correlações positivas entre idade e o Fator $1(\mathrm{p}=0,047)$, significando que quanto maior a idade, maior foi a resiliência Fator 1 (tabela 4). 
Tabela 4. Correlações das variáveis numéricas com escores de resiliência. HC-Unicamp, Campinas-SP, 2013.

\begin{tabular}{|c|c|c|c|c|c|c|c|c|c|c|}
\hline & & Idade & ABVD & AIVD & MEEM & EDG & Resiliência & Fator1 & Fator2 & Fator3 \\
\hline \multirow[t]{3}{*}{ Idade } & $\mathrm{r}=$ & 1 & $-0,056$ & $-0,129$ & $-0,238$ & $-0,193$ & 0,242 & $0,282^{*}$ & 0,066 & 0,129 \\
\hline & $\mathrm{p}=$ & & 0,71 & 0,394 & 0,103 & 0,184 & 0,091 & 0,047 & 0,649 & 0,372 \\
\hline & $\mathrm{n}=$ & 50 & 46 & 46 & 48 & 49 & 50 & 50 & 50 & 50 \\
\hline \multirow[t]{3}{*}{ ABVD } & $r=$ & $-0,056$ & 1 & ,486** & $-0,063$ & $-0,117$ & 0,086 & 0,079 & $-0,022$ & 0,118 \\
\hline & $\mathrm{p}=$ & 0,71 & & 0 & 0,665 & 0,427 & 0,559 & 0,591 & 0,878 & 0,419 \\
\hline & $\mathrm{n}=$ & 46 & 49 & 49 & 49 & 48 & 49 & 49 & 49 & 49 \\
\hline \multirow[t]{3}{*}{ AIVD } & $r=$ & $-0,129$ &, $486 * *$ & 1 &, $326 *$ & $-450 * *$ & 0,195 & 0,236 & 0,08 & 0,074 \\
\hline & $\mathrm{p}=$ & 0,394 & 0 & & 0,022 & 0,001 & 0,179 & 0,103 & 0,587 & 0,612 \\
\hline & $\mathrm{n}=$ & 46 & 49 & 49 & 49 & 48 & 49 & 49 & 49 & 49 \\
\hline \multirow[t]{3}{*}{ MEEM } & $\mathrm{r}=$ & $-0,238$ & $-0,063$ & ,326* & 1 & $-0,089$ & 0,026 & 0,066 & $-0,01$ & $-0,043$ \\
\hline & $\mathrm{p}=$ & 0,103 & 0,665 & 0,022 & & 0,538 & 0,856 & 0,647 & 0,944 & 0,762 \\
\hline & $\mathrm{n}=$ & 48 & 49 & 49 & 51 & 50 & 51 & 51 & 51 & 51 \\
\hline \multirow[t]{3}{*}{ EDG } & $r=$ & $-0,193$ & $-0,117$ &,$- 450 * *$ & $-0,089$ & 1 &,$- 688 * *$ &,$- 703 * *$ &,$- 470 * *$ &,$- 416 * *$ \\
\hline & $\mathrm{p}=$ & 0,184 & 0,427 & 0,001 & 0,538 & & 0 & 0 & 0,001 & 0,002 \\
\hline & $\mathrm{n}=$ & 49 & 48 & 48 & 50 & 51 & 51 & 51 & 51 & 51 \\
\hline \multirow[t]{3}{*}{ Resiliência } & $\mathrm{r}=$ & 0,242 & 0,086 & 0,195 & 0,026 & $-688 * *$ & 1 & ,935** &, $737 * *$ &, $730 * *$ \\
\hline & $\mathrm{p}=$ & 0,091 & 0,559 & 0,179 & 0,856 & 0 & & 0 & 0 & 0 \\
\hline & $\mathrm{n}=$ & 50 & 49 & 49 & 51 & 51 & 57 & 57 & 57 & 57 \\
\hline \multirow[t]{3}{*}{ Fator1 } & $\mathrm{r}=$ &, $282 *$ & 0,079 & 0,236 & 0,066 & $-703 * *$ &, $935 * *$ & 1 &, $589 * *$ &, $474 * *$ \\
\hline & $\mathrm{p}=$ & 0,047 & 0,591 & 0,103 & 0,647 & 0 & 0 & & 0 & 0 \\
\hline & $\mathrm{n}=$ & 50 & 49 & 49 & 51 & 51 & 57 & 57 & 57 & 57 \\
\hline \multirow[t]{3}{*}{ Fator2 } & $\mathrm{r}=$ & 0,066 & $-0,022$ & 0,08 & $-0,01$ & $-470 * *$ &, $737 * *$ &, $589 * *$ & 1 &, $490 * *$ \\
\hline & $\mathrm{p}=$ & 0,649 & 0,878 & 0,587 & 0,944 & 0,001 & 0 & 0 & & 0 \\
\hline & $\mathrm{n}=$ & 50 & 49 & 49 & 51 & 51 & 57 & 57 & 57 & 57 \\
\hline \multirow[t]{3}{*}{ Fator3 } & $\mathrm{r}=$ & 0,129 & 0,118 & 0,074 & $-0,043$ & $-416 * *$ &, $730 * *$ &, $474 * *$ &, $490 * *$ & 1 \\
\hline & $\mathrm{p}=$ & 0,372 & 0,419 & 0,612 & 0,762 & 0,002 & 0 & 0 & 0 & \\
\hline & $\mathrm{n}=$ & 50 & 49 & 49 & 51 & 51 & 57 & 57 & 57 & 57 \\
\hline
\end{tabular}

*Correlação significativa ao nível 0,05

** Correlação significativa ao nível 0,01

$r=$ coeficiente de correlação; $\mathrm{p}=$ valor $\mathrm{p} ; \mathrm{n}=\mathrm{n}^{\circ}$ de participantes 
A análise de regressão linear multivariada evidenciou que a presença de sintomatologia depressiva foi a única variável significativamente associada ao escore de resiliência, evidenciando que idosos com maior número de sintomas depressivos tenderam a apresentar baixos escores em resiliência (tabela 5).

Tabela 5. Análise de regressão logística multivariada para resiliência. HC-Unicamp, Campinas-SP, 2013.

\begin{tabular}{cccc}
\hline Variáveis & p-valor & Beta $^{1}$ & EP $^{2}$ \\
\hline Constante & $<0,001$ & - & 15,479 \\
Genero & 0,848 & $-0,035$ & 2,963 \\
Idade numérica & 0,686 & - & 0,144 \\
Renda mensal & 0,457 & - & 3,557 \\
Escolaridade & 0,448 & $-1,27$ & 1,599 \\
ABVD & 0,764 & - & 0,865 \\
AIVD & 0,539 & $-1,35$ & 0,609 \\
MEEM & 0,661 & - & 0,287 \\
EDG & 0.003 & $-0,596$ & 0,332 \\
\hline
\end{tabular}

Beta $^{1}=$ valor de estimativa ou coeficiente angular (slope)

$\mathrm{EP}^{2}=$ erro padrão

\section{DISCUSSÃO}

Os resultados encontrados neste estudo apontam importantes e significativas associações entre resiliência e funcionalidade. Os idosos com elevada resiliência apresentavam maior número de atividades instrumentais preservadas $(\mathrm{m}=5,2)$, em relação aos de baixa resiliência $(m=3,6)$, o mesmo acontecendo em relação aos sintomas depressivos: idosos com elevada resiliência apresentaram menor sintomatologia depressiva $(\mathrm{m}=2,6) \mathrm{em}$ relação aos idosos com baixa resiliência $(\mathrm{m}=6,4)$. Além disso, a resiliência correlacionou-se negativamente com a sintomatologia depressiva, sendo esta a única variável significativamente associada à resiliência quando se utilizou a análise de regressão multivariada.

A redução de atividades na vida diária se associa comumente à depressão. A limitação funcional, ao lado de outros eventos estressantes, como a perda de pessoas queridas ou de papéis sociais, pode envolver uma ameaça à vida ativa, tornando-se fator de risco para pessoas que valorizam o sucesso pessoal e o controle, contribuindo para um aumento das autoavaliações negativas, particularmente entre aqueles que não estão abertos a novas experiências. ${ }^{23}$

Como importante fator de risco para a depressão, estão as doenças crônicas, que aumentam a vulnerabilidade do idoso à depressão, mais comum entre aqueles com doença coronariana, preditiva de re-hospitalizações e de aumento de incapacidades, ${ }^{24}$ e entre aqueles com hipertensão, diabetes, transtorno de ansiedade e demência. ${ }^{23}$ A sintomatologia depressiva é também elevada entre idosos com artrite reumatoide ou osteoartrite, doença de Parkinson, doença vascular avançada ou infarto. ${ }^{25}$ De um modo geral, idosos que frequentam serviços ambulatoriais são portadores de doenças crônicas, relativo grau de dependência e evidência de sintomas depressivos.

Um estudo descrevendo o perfil desses mesmos idosos em relação às variáveis demográficas e funcionalidade observou que $63,3 \%$ mostravam-se independentes em relação às $\mathrm{ABVD} ; 32,7 \%$ independentes em relação às AIVD; e 32\% dos idosos tinham desenvolvido sintomas depressivos, sendo dependentes tanto 
para ABVD como para AIVD $(\mathrm{p}<0,05) \cdot{ }^{26}$ Esses dados sugerem que idosos que frequentam os serviços ambulatoriais, apesar de sua condição crônica e de um relativo grau de incapacidade funcional, desenvolvem comportamentos resilientes, que os ajudam a resistir a tais adversidades. A resiliência apoia-se em recursos pessoais, como a autoestima, o controle e as competências pessoais ${ }^{8}$ e recursos sociais de enfrentamento, em especial, aqueles que dão continuidade à manutenção dos papéis sociais e à rede de suporte social, ${ }^{27}$ tendo a participação em redes sociais efeito protetor para idosos com e sem demência. ${ }^{28}$

Smith, ${ }^{29}$ investigando a relação entre a resiliência e o desejo por procurar cuidados para sintomas depressivos em 158 idosos da comunidade, afroamericanos, encontrou relação direta e preditiva entre resiliência e o desejo de buscar ajuda, em que os mais resilientes tinham maior facilidade para procurar pelos serviços de saúde mental. A busca por ajuda reflete-se em idosos que frequentam os serviços ambulatoriais do hospital universitário onde se realizou o presente estudo, pois mesmo com condições de transporte pouco favoráveis para os que apresentam limitação funcional, tais idosos frequentam com regularidade os serviços oferecidos, o que, supõe-se, pode refletir uma maior capacidade de lidar com as dificuldades associadas à saúde e funcionalidade.

Quando a resiliência foi avaliada por fatores, encontrou-se elevada e baixa resiliência associadas a maior e menor presença de Fatores 1 e 3, respectivamente. Uma análise dos componentes do Fator $1^{22}$ mostra que estes se associam a ações resolutivas, como por exemplo, "Quando eu faço planos, eu os levo até o fim" e a valores que dão sentido à vida - entre eles, a amizade, a realização pessoal, a satisfação, o ter significados para a vida. Dentro de uma visão humanista, a resiliência é vista como força ou crescimento interno, força pessoal, senso de coerência, capacidade de se pautar por metas de vida e de autotranscendência, abrangendo conteúdos como: sentir-se competente, aceitar a ajuda dos outros, olhar para o lado luminoso da vida, sentir-se o mesmo ao longo da vida e conectar-se com o presente, passado e futuro. ${ }^{30}$ Mais recentemente, tem sido associada às crenças religiosas e espirituais que se associam a melhores resultados em saúde. ${ }^{31}$ Sobreviventes de câncer mais idosos apresentam-se mais resilientes em relação aos mais jovens, apresentando melhores resultados em saúde mental, humor, bem-estar psicológico, bem-estar social e espiritualidade.

$\mathrm{O}$ Fator $3^{22}$ refere-se a itens relativos à autoconfiança e capacidade de adaptação às mudanças, do tipo: "Eu sinto que posso experimentar tempos dificeis porque já experimentei dificuldades antes". Além de itens envolvendo capacidades pessoais, alguns itens englobados pelos Fatores 1 e 3 referemse à capacidade de regular as emoções, capacidade essa que vem sendo apresentada como fator-chave da resiliência (por exemplo, "Eu normalmente posso achar motivo para rir" on "Еu não insisto em coisas com as quais eu não posso fazer nada sobre elas"). A literatura gerontológica tem demonstrado que a regulação emocional se traduz em diminuição do afeto negativo com a idade. ${ }^{32,33}$ Idosos resilientes experimentam menor estresse e reatividade a ele e se recuperam mais rapidamente, ${ }^{34}$ apresentando poucos traços de ansiedade, sintomas depressivos e altos níveis de otimismo. ${ }^{35}$

$\mathrm{O}$ estudo apresentou limites quanto à determinação e tamanho da amostra, por tratarse de uma amostragem de conveniência, o que impede a generalização desses resultados para outras populações. Além disso, o instrumento utilizado para avaliação de resiliência, embora venha sendo utilizado pelos estudos brasileiros, não sofreu adaptação para a população idosa, até o presente momento.

\section{CONCLUSÃO}

A resiliência psicológica mostrou-se associada à preservação da funcionalidade e à menor sintomatologia depressiva, constituindo, portanto, importante fator de proteção para idosos acometidos por doenças crônicas, com relativo grau de dependência e evidência de sintomas depressivos, que caracterizam frequentadores de serviços ambulatoriais. 


\section{REFERÊNCIAS}

1. Frank MH, Rodrigues NL. Depressão, ansiedade, outros distúrbios afetivos e suicídio. In: Freitas EV, Py L, editores. Tratado de geriatria e gerontologia. $2^{\mathrm{a}} \mathrm{ed}$. Rio de Janeiro: Guanabara Koogan; 2006. p. 376-87.

2. Almeida OP, Almeida SA. Confiabilidade da versão brasileira da Escala de Depressão Geriátrica (GDS) versão reduzida. Arq Neuropsiquiatr 1999;57(2B):421-6.

3. Lebrão ML, Laurenti R. Saúde, bem-estar e envelhecimento: o estudo SABE no município de São Paulo. Rev Bras Epidemiol 2005;8(2):127-41.

4. Batistoni SST, Neri AL, Cupertino APFB. Validade da escala de depressão do Center for Epidemiological Studies entre idosos brasileiros. Rev Saúde Pública 2007;41(4):598-605.

5. Rutter M. Psychosocial resilience and protective mechanisms. Am J Orthopsychiatr 2007;57(3):316-31.

6. Wagnild G. Resilience and successful aging: comparison among low and high income older adults. J Gerontol Nurs 2003;29(12):42-9.

7. Staudinger UM, Marsiske M, Baltes P. Resilience and levels of reserve capacity in later adulthood: perspectives from life-span theory. Dev Psychopathol 1993;5(4):541-66.

8. Windle G, Markland DA, Woods RT. Examination of a theoretical model of psychological resilience in older age. Aging Ment Health 2008;12(3):285-92. PubMed; PMID 18728940.

9. Beutel ME, Glaesmer H, Decker O, Fischbeck S, Brahler E. Life satisfaction, distress, and resiliency across the life span of women. Menopause 2009;16(6):1132-8. PubMed; PMID 19543128.

10. Rowe JW, Kahn RL. Human aging: usual and successful. Science 1997;237(4811):143-49.

11. Laranjeira CAS. Do vulnerável ser ao resiliente envelhecer: revisão de literatura. Psicol Teor Pesqui 2007;23(3):327-32.

12. Hildon Z, Montgomery SM, Blane D, Wiggins RD, Netuveli G. Examining resilience of quality of life in the face of health-related and psychosocial adversity at older ages: what is "right" about the way we age? Gerontologist 2010;50(1):36-47. PubMed; PMID 19549715.

13. Mehta M, Whyte E, Lenze E, Hardy S, Roumani Y, Subashan P, et al. Depressive symptoms in late life: associations with apathy, resilience and disability vary between young-old and old-old. Int J Geriatr Psychiatry 2008;23(3):238-43.

14. Hardy S, Contato J, Gill T. Resilience of communitydwelling older persons. J Am Geriatr Soc 2004;52(2):257-62.
15. Folstein MF, Folstein SE, McHugh PR. Mini-mental state: a practical method for grading the cognitive state of patients for the clinician. J Psychiatr Res 1975;12(3):189-98.

16. Lourenço RA, Veras RP. Mini-Exame do Estado Mental: características psicométricas em idosos ambulatoriais. Rev Saúde Pública 2006;40(4):712-9.

17. Bertolucci PHF, Brucki S, Campacci SR, Juliano Y. O Mini-Exame do Estado Mental em uma população geral: impacto da escolaridade. Arq Neuropsiquiatr 1994;52(1):1-7.

18. Katz S, Ford AB, Moskowitz RW, Jackson BA, Jaffe MW. Studies of Illness in the aged the index of ADL: a standardized measure of biological and psychosocial function. JAMA 1963;12:914-9.

19. Lino VTS, Pereira SRM, Camacho LAB, Ribeiro ST Filho, Buksman S. Adaptação transcultural da Escala de Independência em Atividades da Vida Diária (Escala de Katz). Cad Saúde Pública 2008;24(1):103-12.

20. Lawton M, Brody E. Assessment of older people: selfmaintaining and instrumental activities of daily living. Gerontologist 1969;9(3):179-86.

21. Yesavage JA, Brink TL, Terence LR, Lum O, Huang $\mathrm{V}$, Adey M, et al. Development and validation of a geriatric depression screening scale: a preliminary report. J Psychiatr Res 1983;17(1):37-49.

22. Pesce RP, Assis SG, Avanci JQ, Santos NC, Malaquias JV, Carvalhaes R. Adaptação transcultural, confiabilidade e validade da escala de resiliência. Cad Saúde Pública [Internet] 2005 [acesso em 5 jul 2012];21(2):436-48. Disponível em: http://dx.doi. org/10.1590/S0102-311X2005000200010

23. Fiske A, Wetherell JL, Gatz M. Depression in older adults. Annu Rev Clin Psychol 2009;5:363-89.

24. Boyle SH, Williams RB, Mark DB, Brummett BH, Siegler IC, Helms MJ, et al. Hostility as a predictor of survival in patients with coronary artery desease. Psychosom Med 2004;66(5):629-32.

25. Batistoni SST. Depressão. In: Neri AL, Guariento ME, organizadores. Assistência ambulatorial ao idoso. Campinas, SP: Alínea; 2010. p. 235-42.

26. De Paula AFM, Ribeiro LHM, D’Elboux MJ, Guariento ME. Avaliação da capacidade funcional, cognição e sintomatologia depressiva em idosos atendidos em ambulatório de Geriatria. Rev Bras Clin Med 2013;11(3):212-8.

27. Hildon Z, Smith G, Netuveli G, Blane D. Understanding adversity and resilience at older ages. Sociol Health Illn 2008;30(5):726-40. PubMed; PMID 18444953. 
28. McFadden SH, Basting AD. Healthy aging persons and their brains: promoting resilience through creative engagement. Clin Geriatr Med 2010;26(1):149-61. PubMed; PMID 20176299.

29. Smith PR. Resilience: resistance factor for depressive symptom. J Psychiatr Ment Health Nurs 2009;16(9):829-37. PubMed; PMID 19824977.

30. Nygren B, Norberg A, Lundman B. Inner strength as disclosed in narratives of the oldest old. Qual Health Res 2007;17(8):1060-73.

31. Krause N, Bastida E. Religion, suffering, and selfrated health among older Mexican Americans. J Gerontol B Psychol Sci Soc Sci 2011;66(2):207-16. PubMed; PMID 21076086. Pubmed Central; PMCID: 3041974.
32. Kessler EM, Staudinger UM. Affective experience in adulthood and old age: the role of affective arousal and perceived affect regulation. Psychol Aging 2009;24(2):349-62. PubMed; PMID 19485653.

33. Charles ST, Carstensen LL. Social and emotional aging. Annu Rev Psychol 2010;61:383-409. PubMed; PMID 19575618.

34. Montpetit MA, Bergeman CS, Deboeck PR, Tiberio SS, Boker SM. Resilience-as-process: negative affect, stress, and coupled dynamical systems. Psychol Aging 2010;25(3):631-40. PubMed; PMID 20853969.

35. Larcom MJ, Isaacowitz DM. Rapid emotion regulation after mood induction: age and individual differences. J Gerontol B Psychol Sci Soc Sci 2009;64(6):733-41. PubMed; PMID 19808810. Pubmed Central; PMCID 2763016. 